\title{
Design of a wearable cable-driven upper limb exoskeleton based on epicyclic gear trains structure
}

\author{
Feiyun Xiao ${ }^{\mathrm{a}, \mathrm{b}}$, Yongsheng $\mathrm{Gao}^{\mathrm{b}}$, Yong Wang ${ }^{\mathrm{a}, *}$, Yanhe Zhu ${ }^{\mathrm{b}}$ and Jie Zhao ${ }^{\mathrm{b}}$ \\ ${ }^{a}$ School of Mechanical Engineering, Hefei University of Technology, Hefei, Anhui, China \\ ${ }^{\mathrm{b}}$ State Key Laboratory of Robotics and System, Harbin Institute of Technology, Harbin, Heilongjiang, \\ China
}

\begin{abstract}
.
BACKGROUND: Many countries, including Japan, Italy, and China are experiencing demographic shifts as their populations age. Some basic activities of daily living (ADLs) are difficult for elderly people to complete independently due to declines in motor function.

OBJECTIVE: In this paper, a 6-DOF wearable cable-driven upper limb exoskeleton (CABexo) based on epicyclic gear trains structure is proposed.

METHODS: The main structure of the exoskeleton system is composed of three epicyclic gear train sections. This new exoskeleton has a parallel mechanical structure to the traditional serial structure, but is stiffer and has a stronger carrying capacity. The traditional gear transmission structure is replaced with a cable transmission system, which is quieter, and has higher accuracy and smoother transmission.

RESULTS AND CONCLUSIONS: The static workspace of the exoskeleton is large enough to meet the demand of assisting aged and disabled individuals in completing most of their activities of daily living (ADLs).
\end{abstract}

Keywords: Epicyclic gear train, cable-driven, upper limb exoskeleton, pre-tensioner

\section{Introduction}

Many countries, including Japan, Italy, and China are experiencing demographic shifts as their populations age [1]. Some basic activities of daily living (ADLs) are difficult for elderly people to complete independently due to declines in motor function. Researchers are developing wearable exoskeleton robots to help the aged and disabled with completing ADLs [2-9]. Ideally, wearable exoskeleton should be ergonomical, light-weight, highly reliable and accurate, very stiff, and inexpensive [2].

Many researchers have been investigating and developing a variety of types of wearable exoskeletons. Sugar et al. [2] developed a wearable exoskeleton robot named RUPERT to assist with the rehabilitation of arm functions, after a loss of function. RUPERT is actuated by pneumatic muscles through a serial link mechanism. Martinez et al. [3] designed a five degree of freedoms (DOF) upper limb exoskeleton

\footnotetext{
${ }^{*}$ Corresponding author: Yong Wang, School of Mechanical Engineering, Hefei University of Technology (HFUT), Hefei 230009, Anhui, China. Tel.: +86 13856097243; Fax: +86 045186413392; E-mail: simenkouwang@ sina.com.
}

0928-7329/17/\$35.00 (c) 2017 - IOS Press and the authors. All rights reserved

This article is published online with Open Access and distributed under the terms of the Creative Commons Attribution NonCommercial License (CC-BY-NC 4.0). 
actuated by DC motors and pneumatic muscles; its actuators were placed on an external base to decrease the mass and inertia of the exoskeleton by transmitting power via cables. Although the pneumatic muscle can provide flexible driving torques, these wearable exoskeleton systems actuated by pneumatic muscles with air cylinder may be difficult for patients to carry. Serea et al. [4] developed a hybrid FES-Electric upper arm wearable exoskeleton. However, FES creates muscle fatigue problems that have not yet been solved [5]. Most of these exoskeletons have a serial structure, which is easy to control and has a large workspace. However, each DOF of the serial link mechanism is driven by a single link, which makes the serial mechanism less stiff when compared with the parallel mechanism. This results in the carrying capacity of serial mechanism being weaker than in the parallel mechanism.

The cable-driven mechanism allows the system to be quieter and have high accuracy and smooth transmissions which are necessary for wearable exoskeletons [6-9]. Mao et al. [6,7] developed a cable driven arm exoskeleton (CAREX) for upper arm rehabilitation and Shao et al. [8] designed a 3-DOF cable-driven upper arm exoskeleton. However, the cable-driven parallel mechanism with flexible links is hard to be controlled. Ball et al. [9], proposed a traditional serial structure 5-DOF rehabilitation robot with the driving energy transmitted by cables. It can be controlled easily, however, it still exhibits the shortcomings of serial mechanism.

To overcome these problems, a cable-driven upper limb wearable exoskeleton based on epicyclic gear trains is proposed in this paper. This new structure has 6 DOFs, a three epicyclic gear train, and sections that compose the exoskeleton system. The revolution motion and the spinning motion of the planet wheel forms a 2-DOF coupled motion produced by the driving wheel and the driving link. The upper arm, forearm, and palm of the user's upper limb are driven by corresponding planet wheels, and each planet wheel is supported and driven by two parts, which is similar to the parallel mechanism. The traditional gear transmission structure is replaced with a cable transmission system. The principle of the cable transmission system is the same as the gear transmission structure. However, it does not transmit torque using the meshing force between gears, rather, it uses cables on wheel faces which results in a quiet process, higher accuracy, and smoother transmission. The main body of the exoskeleton is rigid which can be controlled more easily than the parallel mechanism with flexible links. Further, we design a pre-tensioner to ensure the reliability of cable-driven mechanisms.

\section{Mechanical design}

\subsection{DOF selection and Denavit-Hartenberg parameters}

The shoulder internal/external rotation DOF is used less frequently than other DOFs [2]. The exosystem is composed of three epicyclic gear trains sections, ignoring the shoulder internal/external rotation DOF. As it has a large workspace, the exoskeleton could assist those with physical disabilities with most of the movements associated with ADLs as illustrated in Fig. 7 in section three. The model of the exoskeleton is shown in Fig. 1. All DC motors are placed on the backboard with an additional forces transmission system adapted from [3].

The Denavit-Hartenberg parameters of the exoskeleton listed in Table 1 can be obtained using the connecting rod coordinates established in Fig. 1 . The range of the variable is based on Table 1. Here, $l_{2}$ is the length of upper arm, $d_{4}$ is the length of the forearm, and $l_{6}$ is the distance between the wrist joint and the end point. 
Table 1

The Denavit-Hartenberg parameters of the exoskeleton

\begin{tabular}{cccccc}
\hline Link $i$ & Variable $\theta_{i}$ & $\alpha_{i}$ & $l_{i}$ & $d_{i}$ & Range of variable $\theta_{i}$ \\
\hline 1 & $\theta_{1}$ & $-90^{\circ}$ & 0 & 0 & $-140^{\circ} \sim 90^{\circ}$ \\
2 & $\theta_{2}$ & $90^{\circ}$ & $l_{2}$ & 0 & $-180^{\circ} \sim 40^{\circ}$ \\
3 & $\theta_{3}$ & $90^{\circ}$ & 0 & 0 & $90^{\circ} \sim 230^{\circ}$ \\
4 & $\theta_{4}$ & $-90^{\circ}$ & 0 & $d_{4}$ & $-90^{\circ} \sim 90^{\circ}$ \\
5 & $\theta_{5}$ & $90^{\circ}$ & 0 & 0 & $-117^{\circ} \sim-63^{\circ}$ \\
6 & $\theta_{6}$ & $0^{\circ}$ & $l_{6}$ & 0 & $-73^{\circ} \sim 70^{\circ}$ \\
\hline
\end{tabular}

Table 2

Comparison between CABexo and existing exoskeleton systems

\begin{tabular}{lclll}
\hline Exoskeleton systems & DOF & Driving type & Configuration & Motor arrangement \\
\hline CABexo & 6 & DC motor(cable) & Similar parallel & Backboard \\
ARMin [13] & 6 & DC motor & Series & Corresponding joint \\
RUPERT III [2] & 5 & Pneumatic muscle & Series & Corresponding joint \\
CAREX [9] & 7 & DC motor(cable) & Parallel & Backboard \\
IKO [3] & 5 & DC motor & Series & Backboard \\
\hline
\end{tabular}

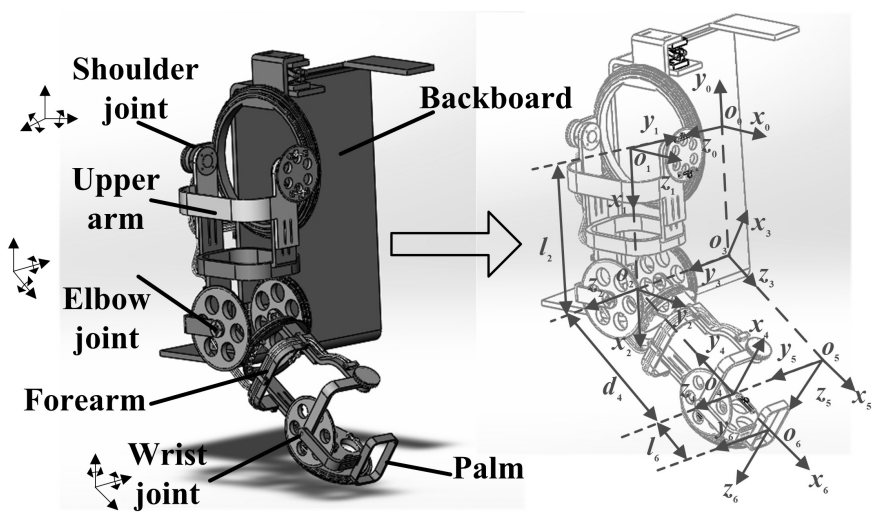

Fig. 1. The 6-DOF upper limb exoskeleton model.

\subsection{The shoulder joint and the wrist joint parts of the exoskeleton}

The shoulder joint part of the exoskeleton driving upper arm rotates with the shoulder joint and has two DOFs - the shoulder flexion/extension DOF and the shoulder abduction/adduction DOF. The schematic diagram of this part is shown in Fig. 2a and the structural diagram is illustrated in Fig. 2b. The radiuses of the driving wheel $b$ and planet wheel $c$ are $R_{b}$ and $R_{c}$, respectively. The angular velocities of the driving link, driving wheel, and planet wheel are $w_{a}, w_{b}$ and $w_{c}$, respectively. The actual motion of the planet wheel is generated by the driving link and the driving wheel, which is composed of the revolution motion $w_{c x}$ and the spinning motion $w_{c y}$. The angular velocity of the planet wheel relative to the driving link is represented by $w_{c}^{a}$, which is equal to $w_{c y}$. It is clear that $w_{c x}$ is equal to $w_{a}$. This can be expressed in the following formula:

$$
w_{c x}=w_{a}, w_{c y}=w_{c}^{a}
$$

To obtain $w_{c}^{a}$, we can virtually add a motion $-w_{a}$ which is in the opposite direction of the driving wheel of this epicyclic gear train. Then, the absolute linear velocity of point $D$ on the planet wheel $c$ 


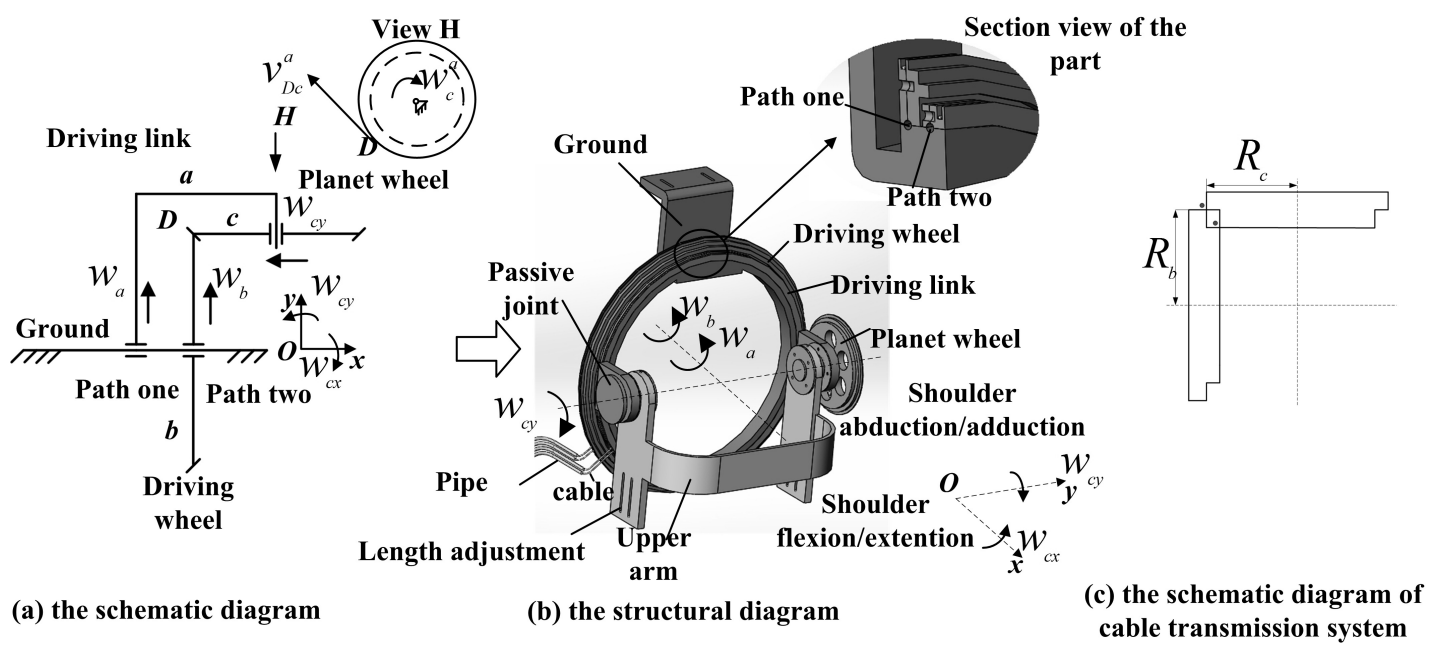

Fig. 2. The shoulder joint part of exoskeleton.

represented by $v_{D c}^{a}$ should be equal to the absolute linear velocity of point $D$ on the driving wheel $b$ represented by $v_{D b}^{a}$. This allows the angular velocity of planet wheel relative to the driving link to be easily obtained. Then, Eq. (1) can be re-expressed as Eq. (3).

$$
\begin{aligned}
& v_{D c}^{a}=v_{D b}^{a}=R_{b}\left(w_{b}-w_{a}\right), w_{c}^{a}=v_{D c}^{a} / R_{c} \\
& w_{c x}=w_{a}, w_{c y}=w_{c}^{a}=R_{b}\left(w_{b}-w_{a}\right) / R_{c}
\end{aligned}
$$

If the direction $w_{a}$ or $w_{b}$ is in the opposite direction from that shown in Fig. 2a, the value of $w_{a}$ or $w_{b}$ in Eq. (3) is negative, and the direction of $w_{c y}$ in view $H$ will be clockwise if the value of $w_{c}^{a}$ in Eq. (3) is positive.

The traditional gear transmission structure is replaced with cable transmission system as shown in Fig. 2c. As the cable is a flexible one-way transmission body, each DOF of the cable-driven mechanism can be accomplished by two cables placed on a wheel with a phase difference of 180 degrees. The driving wheel and the planet wheel should be two-stage as shown in Fig. 2c.

As it is hard to process large rolling bearings, we adopt a similar structure as shown in the sectioned view of the part in Fig. 2b. Steel balls with the same diameter, are placed in path one and path two; the steel balls function as simplified rolling bearings. The driving wheel and the driving link are each divided into two parts making it convenient to place the steel balls.

The wrist joint part of the exoskeleton is similar to the shoulder part. It has two DOFs - the wrist flexion/extension DOF and the wrist radial deviation/ulnar deviation DOF. A detailed diagram of the wrist joint part of exoskeleton is shown in Fig. 3. The angular speeds of the revolution and rotation of the planet wheel can be obtained by Eq. (3).

\subsection{The elbow joint part of the exoskeleton}

The elbow joint part of the exoskeleton has two DOFs: the elbow flexion/extension and the forearm supination/pronation. The schematic diagram of the elbow joint part of exoskeleton is shown in Fig. 4. The motion of the planet wheel driving the forearm of the user to rotate with the elbow joint is controlled by two driving wheels. The radiuses of the driving wheel $a, b$, and planet wheel $c$ are $R_{a}, R_{b}$ and 


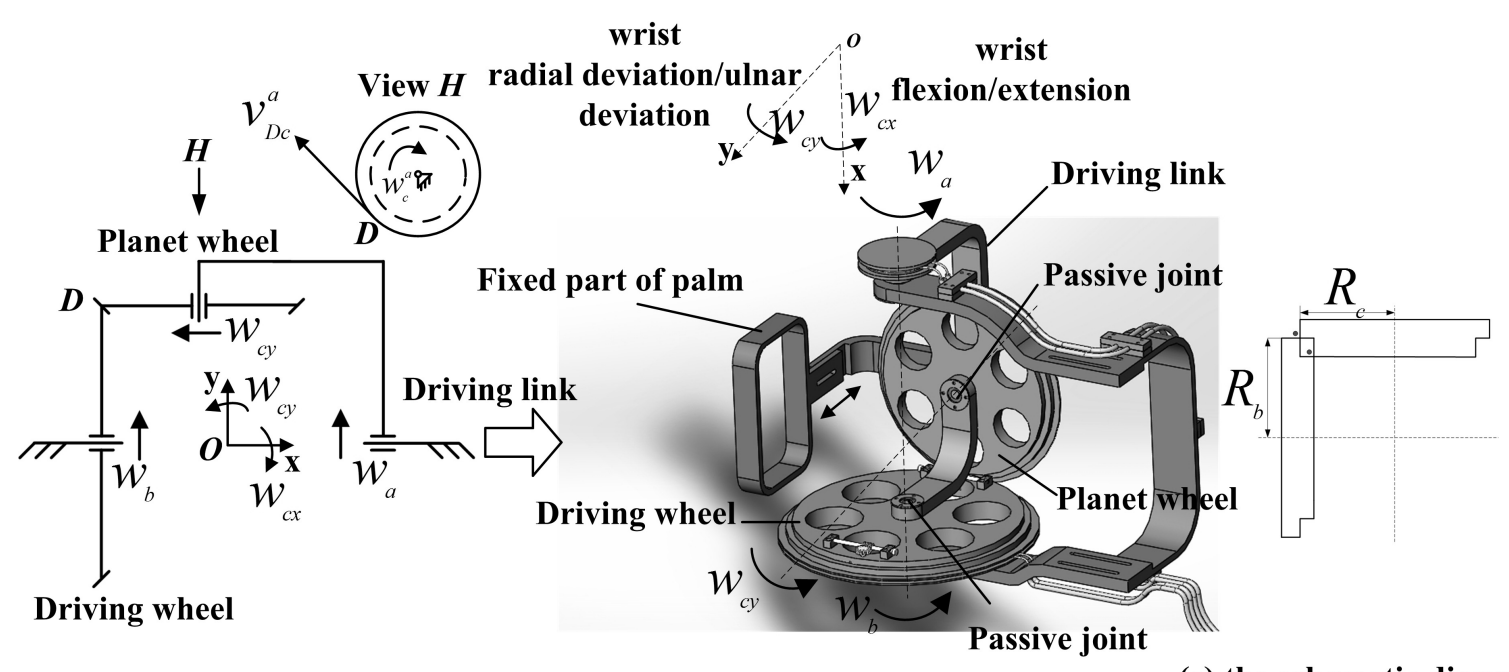

(a) the schematic diagram

(b) the structural diagram

(c) the schematic diagram of cable transmission system

Fig. 3. The wrist joint part of the exoskeleton.

$R_{c}$, respectively, and the angular velocities are $w_{a}, w_{b}$, and $w_{c}$, respectively. The angular velocities of the planet wheel relative to the driving wheels $a$ and $b$ are represented by $w_{c}^{a}$ and $w_{c}^{b}$, respectively. According to the angular velocity composition theorem of intersecting axes, we can obtain the following relationships in Eq. (4). By projecting the vector equation Eq. (4) to the $x$ axis and $y$ axis, $w_{c x}$ and $w_{c y}$ can be obtained based on Figs 4 and 5. Below, the situation presented in Fig. 5a is used as an example:

$$
\begin{aligned}
& \overrightarrow{w_{c}}=\overrightarrow{w_{a}}+\overrightarrow{w_{c}^{a}}=\overrightarrow{w_{b}}+\overrightarrow{w_{c}^{b}} \\
& w_{c x}=w_{a}-w_{c}^{a} \cos \delta_{1}=w_{b}-w_{c}^{b} \cos \delta_{3}, w_{c y}=0+w_{c}^{a} \sin \delta_{1}=0-w_{c}^{b} \sin \delta_{3}
\end{aligned}
$$

According to Fig. $4 \mathrm{a}$ and $\mathrm{c}$, the relationship between $\delta_{1}, \delta_{3}$ and $R_{a}, R_{b}, R_{c}$ can be expressed as follows:

$$
R_{a}=R_{b}, \delta_{1}=\delta_{3}, \cot \delta_{1}=\cot \delta_{3}=R_{c} / R_{a}
$$

Then, Eq. (5) can be re-expressed as follows:

$$
w_{c x}=\left(w_{a}+w_{b}\right) / 2, w_{c y}=R_{a}\left(w_{a}-w_{b}\right) / 2 R_{c}
$$

In the situations illustrated in Fig. 5b, $\mathrm{c}$ and d, $w_{c x}$ and $w_{c y}$ can also be obtained by Eq. (7). In Eq. (7), if the direction of $w_{a}$ or $w_{b}$ is opposite from the direction shown in Fig. 4a, the value of $w_{a}$ or $w_{b}$ is negative. The direction of $w_{c y}$ in view $H$ will be clockwise if the value of $w_{c y}$ is positive and the direction of $w_{c x}$ will be the same as $w_{a}$, as shown in Fig. $4 \mathrm{a}$, if $w_{c x}$ is positive. The driving wheels and the planet wheel are in four-stages with incremental diameters as shown in Fig. 4c.

\subsection{The pre-tensioner of the cable-driven mechanism}

To ensure the reliability of the cable-driven mechanisms, the pre-tensioner should be examined. In this paper, the worm and worm wheel structure have been used as the pre-tensioner, which makes use of its self-locking characteristics [10]. When the lead angle of worm $\gamma$ is less than the equivalent frictional angle $\varphi$, the worm can drive the worm wheel, but the opposite is not possible. The shoulder part is taken as an example illustrated in Fig. 6. 


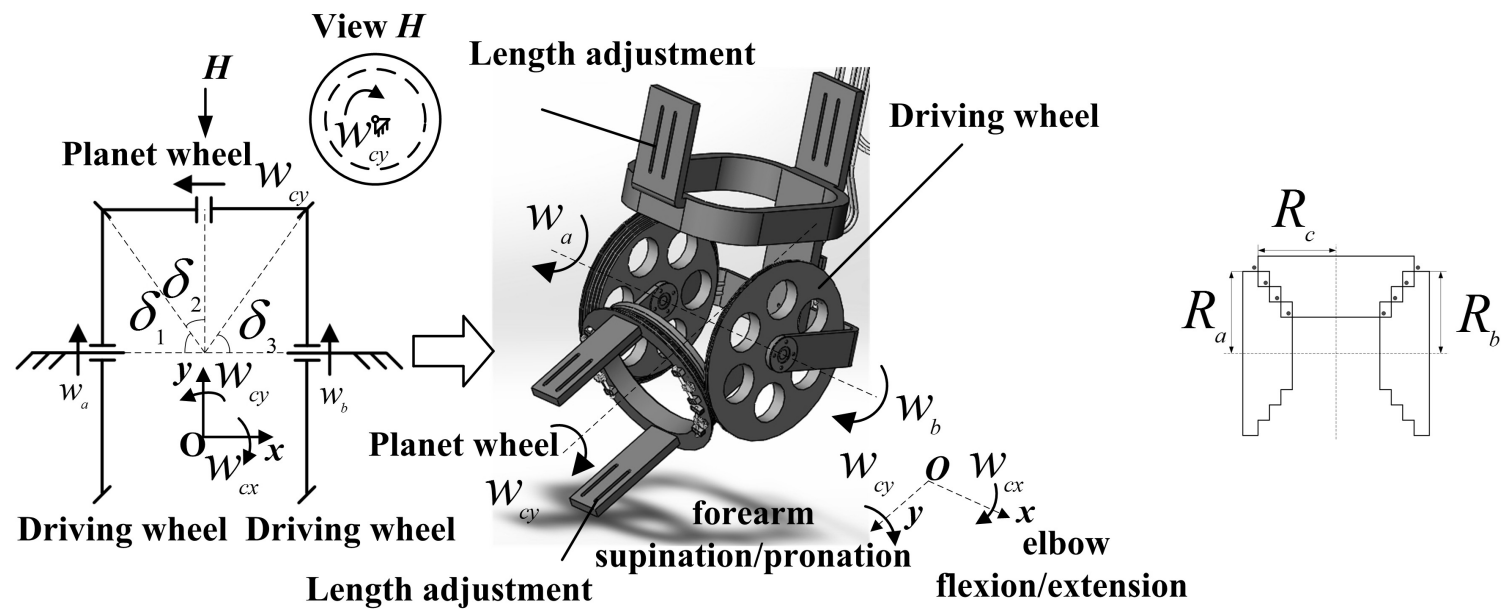
(a) the schematic diagram
(b) the structural diagram
(c) the schematic diagram of cable transmission system

Fig. 4. The elbow joint part of the exoskeleton.

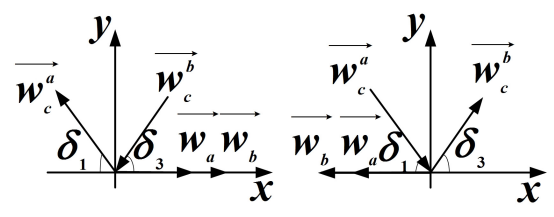

$\boldsymbol{w}_{a}>0, \boldsymbol{w}_{b}>0$

(a) $\boldsymbol{w}_{a}<0, \boldsymbol{w}_{b}<0$

(b)

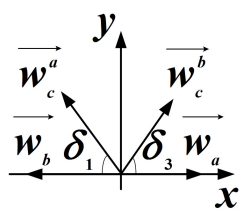

$\boldsymbol{w}_{a}>0, \boldsymbol{w}_{b}<0$

(c)

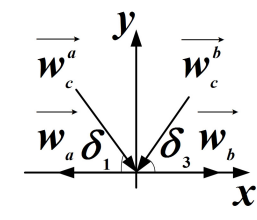

$\boldsymbol{w}_{a}<0, \boldsymbol{w}_{b}>0$

Fig. 5. The projection of the angular velocities.

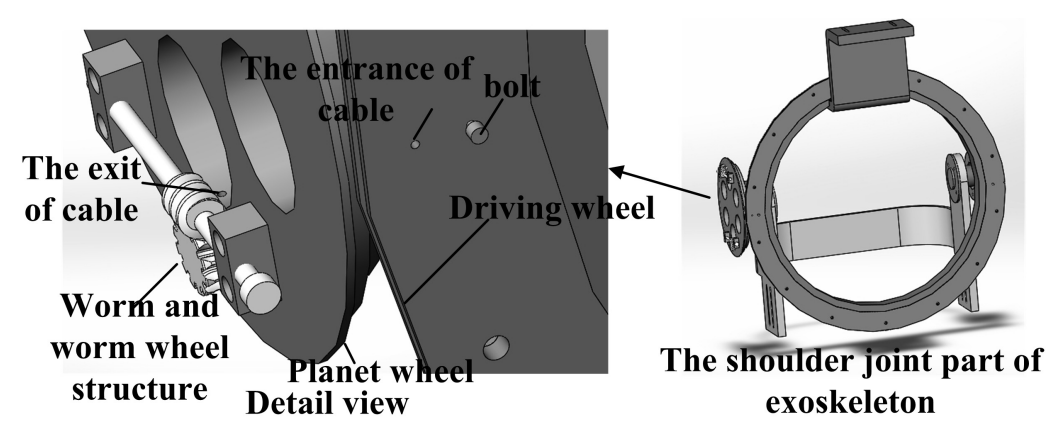

Fig. 6. The arrangement of pre-tensioner.

\section{Forward kinematics and workspace}

According to Fig. 1 and Table 1, the transformation matrixes can be obtained using the following equation:

$$
{ }^{0} T_{6}={ }^{0} A_{1}{ }^{1} A_{2}{ }^{2} A_{3}{ }^{3} A_{4}{ }^{4} A_{5}{ }^{5} A_{6}
$$



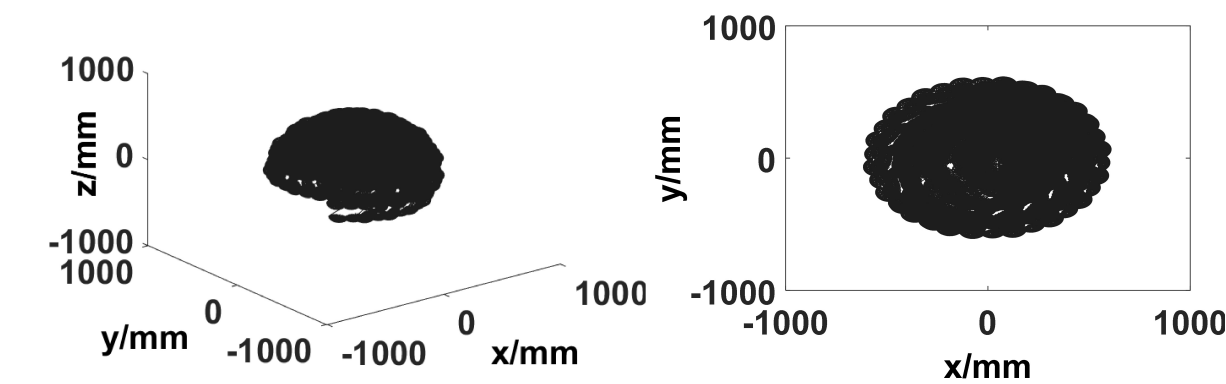

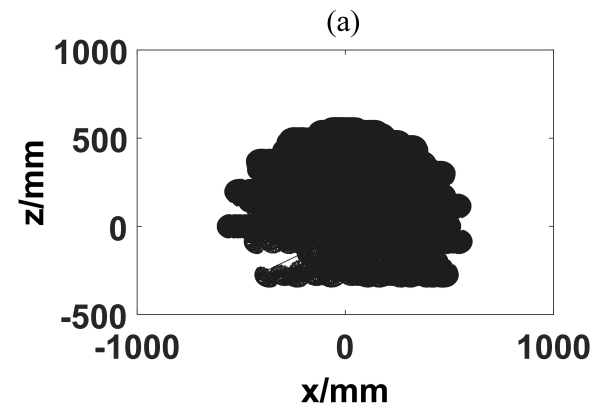

(c)

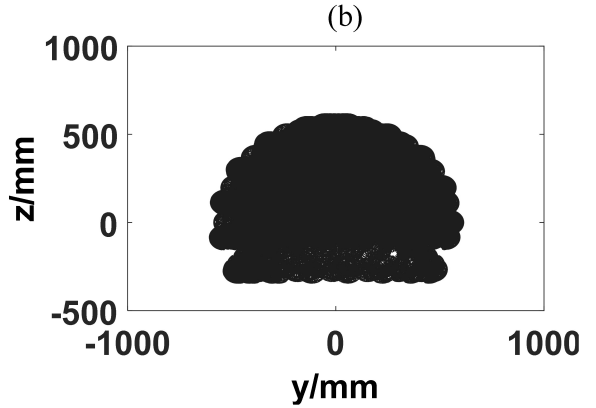

(d)

Fig. 7. The workspace of the exoskeleton.

$$
=\left[\begin{array}{llll}
c \theta_{1} & 0 & -s \theta_{1} & 0 \\
s \theta_{1} & 0 & c \theta_{1} & 0 \\
0 & -1 & 0 & 0 \\
0 & 0 & 0 & 1
\end{array}\right]\left[\begin{array}{llll}
c \theta_{2} & 0 & s \theta_{2} & l_{2} c \theta_{2} \\
s \theta_{2} & 0 & -c \theta_{2} & l_{2} s \theta_{2} \\
0 & 1 & 0 & 0 \\
0 & 0 & 0 & 1
\end{array}\right] \cdots\left[\begin{array}{llll}
c \theta_{6} & -s \theta_{6} & 0 & l_{6} c \theta_{6} \\
s \theta_{6} & c \theta_{6} & 0 & l_{6} s \theta_{6} \\
0 & 1 & 1 & 0 \\
0 & 0 & 0 & 1
\end{array}\right]
$$

Where $s \theta_{i}$ and $c \theta_{i}$ represent sine of $\theta_{i}$ and cosine of $\theta_{i}$, respectively. $l_{2}$ is the length of the upper arm, $d_{4}$ is the length of forearm, and $l_{6}$ is the distance between the wrist joint and the end point. The workspace of the exoskeleton illustrated in Fig. 7 can be obtained using Eq. (8), and the static workspace of the exoskeleton is reasonably large in order to meet the demands of assisting the aged and disabled to complete ADLs.

\section{Analysis of the transmission characteristics of the cable transmission system}

In this section, the transmission characteristics of the cable transmission system are compared with the traditional gear transmission structure. The angle of transmission backlash is a primary indicator reflecting transmission characteristics, which is well if the angle of transmission backlash is small enough $[11,12]$. The angle of transmission backlash of the cable transmission system is obtained as follows:

$$
\begin{aligned}
& \theta_{c b}=2 T_{\text {preload }} T_{\text {load }}^{2}\left(r_{1}+r_{2}\right) / \mu r_{2} G\left(T_{\text {preload }}^{2}-T_{\text {load }}^{2}\right) \\
& \theta_{g b}=\arctan \left[\left(\left|E_{s s 1}+E_{s s 2}\right|+T_{s 1}+T_{s 2}+E_{s \Delta 1}+E_{s \Delta 2}\right) \cos \left(\alpha_{n}\right) / r_{2}\right]
\end{aligned}
$$

Where $\theta_{c b}$ and $\theta_{g b}$ represent the angles of transmission backlash for the cable transmission system and the gear transmission system respectively. $T_{\text {load }}$ is the load force, $T_{\text {preload }}$ is the preload force, $r_{1}$ is the radius of the driving wheel, $r_{2}$ is the radius of driven wheel, $G$ is the tension rigidity, and $\mu$ represents 


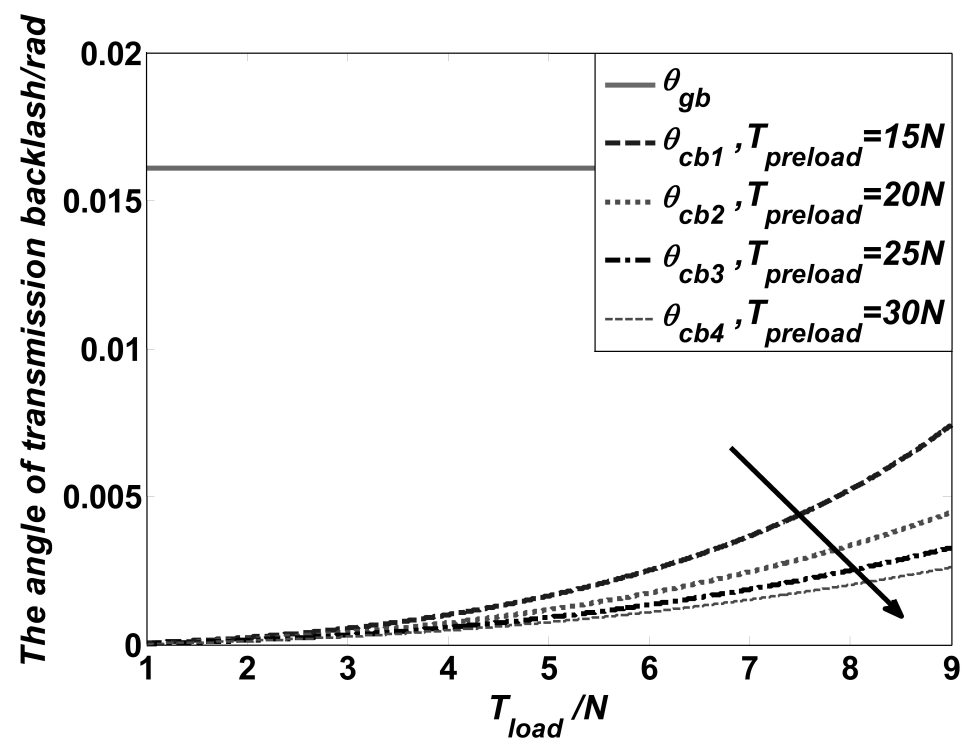

Fig. 8. The angle of transmission backlash.

the friction coefficient. $E_{s s 1}$ and $E_{s s 2}$ are the upper deviation of tooth thickness of the driving wheel and driven wheel. $T_{s 1}$ and $T_{s 2}$ are the tolerance of tooth thickness of the driving wheel and driven wheel. $E_{s \Delta 1}$ and $E_{s \Delta 2}$ are the compensation parts of the manufacturing error of the driving wheel and driven wheel. $\alpha_{n}$ is the normal pressure angle.

If the wrist joint part is taken as an example, the value of some of the parameters are chosen as follows: $T_{\text {load }}$ is a variable, and other parameters can be obtained by referring to related tables in [13]. Then, the comparison of $\theta_{c b}$ and $\theta_{g b}$ is shown in Fig. 8. The results show that $\theta_{c b}$ is much less than $\theta_{g b}$ and $\theta_{c b}$ decreases when the preload force increases, which means that the cable transmission system has better accuracy than the traditional gear transmission system. The cable transmission system transmits energy by static friction between the cable and wheel avoiding oscillation, which exists in the traditional gear transmission system on account of the gear meshing effect; therefore, the cable transmission system is quieter and has smoother transmission than the traditional gear transmission system.

\section{Discussion and conclusion}

In this paper, a newly-designed cable-driven upper limb exoskeleton (CABexo) based on epicyclic gear trains is described. Comparisons between CABexo and some of the existing exoskeleton systems show that it is able to meet the movement needs of most disabled and elderly individuals. As CABexo is a cable-driven exoskeleton, the transmission system is smoother and more stable than ARMin, RUPERT III, and IKO. The revolution motion and the spinning motion of the planet wheel form a 2-DOF coupled motion in CABexo. The upper arm, forearm, and palm of the user's upper limb are driven by corresponding planet wheels, and, in a parallel mechanism, each planet wheel is supported and driven by two parts. Although the CAREX exoskeleton system is a cable-driven system, it is harder to control than CABexo with a parallel mechanism. In CABexo, all DC motors are placed on the backboard with an additional force transmission system, which makes the weight supported by the user's upper limb 
lighter than ARMin and RUPERT III. Additionally, a pre-tensioner based on the self-locking characteristics of the worm and worm wheel structure is considered in order to ensure the reliability of the cable-driven mechanisms in CABexo. Future work will optimize the transmission forces system and design a reasonable control system.

\section{Acknowledgments}

The work reported in this paper is supported by the Nuclear Science Foundation of China (Grant No. 2015BAF01B03).

\section{Conflict of interest}

None to report.

\section{References}

[1] Luy M, Flandorfer P and Giulio PD. Ageing in an aged society: experiences and attitudes of Catholic order members towards population ageing and older people. Ageing and Society. 2015; 35(1): 1. doi: 101017/S0144686X13000421.

[2] Sugar TG, He J et al. Design and control of RUPERT: a device for robotic upper extremity repetitive therapy. IEEE Transactions on Neural Systems and Rehabilitation Engineering. 2007; 15(3): 336. doi: 101109/TNSRE.2007.903903.

[3] Martinez F, Retolaza I et al. Design of a five actuated DOF upper limb exoskeleton oriented to workplace help. Proceedings of the 2nd; Biennial IEEE/RAS-EMBS International Conference on Biomedical Robotics and Biomechatronics; 2008. doi: 101109/BIOROB.2008.4762788.

[4] Serea F, Poboroniuc M et al. Preliminary tests on a hybrid upper arm exoskeleton for upper arm rehabilitation for disabled patients. 2014 International Conference and Exposition on Electrical and Power Engineering (EPE 2014); 2014.

[5] Antonio J et al. Online assessment of human-robot interaction for hybrid control of walking. Sensors. 2012; 12: 215. doi: 103390/s120100215.

[6] Mao Y, Agrawal SK et al. Design of a cable-driven arm exoskeleton (CAREX) for neural rehabilitation. IEEE Transactions on Robotics. 2012; 28(4): 922. doi: 101109/TRO.2012.2189496.

[7] Mao Y, Jin X et al. Human movement training with a cable driven arm exoskeleton (CAREX). IEE Transactions on Neural Systems and Rehabilitation Engineering. 2015; 23(1): 84. doi: 101109/TNSRE.2014.2329018.

[8] Shao ZF, Tang X et al. Optimal design of a 3-DOF cable-driven upper arm exoskeleton. Advances in Mechanical Engineering. 2014; 1. doi: 101155/2014/157096.

[9] Ball SJ, Brown IE et al. MEDARM: a rehabilitation robot with 5DOF at the shoulder complex. 2007 IEEE/ASME International Conference on Advanced Intelligent Mechatronics; 2007.

[10] Sun H, Chen Z et al. Theory of Machines and Mechanisms (The $7^{\text {th }}$ edition). High Education Press. Beijing, China; 2006.

[11] Wang W et al. Machine design handbook (New edition). China Machine Press, Beijing, China; 2004.

[12] Lu Y. Study on the principle and design method of the precise cable drive, PhD. Dissertation, National University of Defense Technology; 2013.

[13] Reinkensmeyer DJ, Kahn LE, Averbuch M et al. Understanding and treating arm movement impairment after chronic brain injury: Progress with the ARM Guide. Journal of Rehabilitation Research and Development. 2000; 37: 653. 